\title{
Renal Dysfunction following Direct Current Cardioversion of Atrial Fibrillation: Incidence and Risk Factors
}

\author{
Nicolai Grüner-Hegge ${ }^{a}$ Danesh K. Kella ${ }^{b}$ Deepak Padmanabhan ${ }^{b}$ \\ Abhishek J. Deshmukh ${ }^{b}$ Ramila Mehta ${ }^{c}$ David Hodge ${ }^{c}$ Rowlens M. Melduni ${ }^{b}$ \\ Eddie L. Greene ${ }^{d}$ Paul A. Friedman ${ }^{b}$ \\ ${ }^{a}$ Cambridge University, Cambridge, UK; ${ }^{b}$ Division of Cardiovascular Diseases, Mayo Clinic, Rochester, MN, USA; \\ 'Division of Biomedical Statistics and Informatics, Mayo Clinic, Jacksonville, FL, USA; ${ }^{\mathrm{d}}$ Division of Nephrology and \\ Hypertension, Department of Internal Medicine, Mayo Clinic, Rochester, MN, USA
}

\section{Keywords}

Acute kidney injury · Atrial fibrillation · Direct current cardioversion

\begin{abstract}
Introduction: Emerging data suggest that cardioversion for atrial fibrillation (AF) may be associated with acute kidney injury (AKI). However, limited data are available regarding the incidence and risk factors for AKI after direct current cardioversion (DCCV) of AF. Methods: All patients undergoing DCCV at Mayo Clinic between 2001 and 2012 for AF were prospectively enrolled in a database. All patients with serum creatinine (SCR) values pre- and post-cardioversion were reviewed for AKI, defined as a $\geq 25 \%$ decline in eGFR (estimated glomerular filtration rate) from baseline value within 7 days of the DCCV. Results: Of the 6,427 eligible patients, 1,256 (19.5\%) patients had pre- and post-DCCV SCR available and formed the cohort under study. The mean age was 70.4 (SD $11.7)$ years, and $67.3 \%$ were male. During the study period, 131 (10.4\%) patients suffered from AKI following DCCV. AKI was independently associated with inpatient status (OR 26.79; 95\% Cl 3.69-194.52), $\mathrm{CHA}_{2} \mathrm{DS}_{2}$-VASc score (OR 1.25;
\end{abstract}

95\% Cl 1.11-1.41), prior use of diuretics (OR 1.59; 95\% Cl 1.03-2.46), and absence of CKD (OR 1.61; 95\% Cl 1.04-2.49), and was independent of the success of the DCCV. None of the patients required acute dialysis during the study outcome period. Conclusion: AKI following DCCV of AF is common, self-limited, and without the need for replacement therapies.

(c) 2020 S. Karger AG, Basel

\section{Introduction}

Atrial fibrillation (AF) is the most common arrhythmia, with an estimated prevalence predicted to increase to 12.1 million by 2030 [1]. Management of this rhythm disorder focuses on restoration and maintenance of sinus rhythm, rate control, and mitigation of risk of stroke [2]. Despite no significant mortality benefit of rhythm control in AF when compared to rate control, direct current cardioversion (DCCV) remains the cornerstone to restore sinus rhythm acutely in patients with symptomatic AF for the last five decades [3-5]. 
Previously, thromboembolic and arrhythmic complications associated with DCCV of AF have been described in detail, but there is a scarcity of data on incidence of renal dysfunction after DCCV of AF [6-8]. Traditional risk factors of AF such as advanced age, hypertension, diabetes mellitus, coronary artery disease and congestive heart failure often coexist and may predispose to the development of kidney disease [9-11]. AF has been associated with chronic kidney disease and a decline in renal function [12]. Moreover, impairment of the renin-angiotensin-aldosterone axis, one of the central regulators of renal blood flow may be a predisposing factor $[13,14]$.

The present study aimed to investigate the incidence and predictors of acute kidney injury (AKI) following DCCV for AF in a large cohort of consecutive patients.

\section{Methods}

\section{Study Group}

All patients' $\geq 18$ years of age who underwent elective inpatient or outpatient DCCV of atrial arrhythmias at the Mayo Clinic Rochester, MN, between January 2001 and December 2012 were prospectively enrolled in a registry. Demographics and clinical data were collected and entered in the database during the study period. This dataset was queried to select patients with serum creatinine (SCR) available 90 days before the DCCV to establish baseline and post-DCCV SCR available within 90 days after the event to assess for the outcome. The latest heart rate (HR) recorded in the medical records immediately before the DCCV was used as the "pre-DCCV HR," and the latest HR recorded before discharge from the DCCV suite from the continuous telemetry or 12-lead EKG was recorded as the "post-DCCV HR." The most recent medications used before the DCCV were recorded in the database.

\section{Cardioversion Details}

All DCCV were performed electively in a dedicated cardioversion suite under the supervision of a physician. The DCCV protocol has been previously described in detail [15]. Briefly, defibrillator pads were applied to the right of the sternum and the left scapula. Synchronized cardioversion was performed at $50 \mathrm{~J}$ and increased to a maximum of $360 \mathrm{~J}$. If atrial arrhythmia recurred within the 5 min of initial success, another shock at the previous level was delivered. The procedure was deemed successful if sinus rhythm was maintained for greater than $30 \mathrm{~min}$. A 12-lead electrocardiogram was obtained at the end of successful cardioversion. The use of transesophageal echocardiogram guidance to evaluate for a left atrial thrombus and sedation was at the discretion of the performing physician. All patients received anticoagulation for at least 4 weeks in the form of warfarin with goal INR of 2.0-3.0, IV heparin, LMWH, or a direct oral anticoagulant. Parental or subcutaneous anticoagulant for bridging was used as needed for patients discharged on warfarin therapy.

\section{Outcomes}

The primary outcome of the study was AKI, defined as a $\geq 25 \%$ decline in eGFR (estimated glomerular filtration rate) from base- line value within 7 days of the DCCV based on the RIFLE (Risk, Injury, Failure, Loss of kidney function, and End-stage kidney disease) classification [16]. The latest SCR available prior to the DCCV was used as the baseline value. eGFR was calculated using the Modification of Diet in Renal Disease (MDRD) Study equation [17]. The highest value of SCR available within 7 days after the DCCV was used to determine the primary outcome. The secondary outcome was defined as the need for short-term or long-term hemodialysis within 90 days of the DCCV.

\section{Inclusion and Exclusion Criteria}

We excluded all patients with a prior history of hemodialysis or ESRD or those who underwent DCCV under emergent circumstances. We excluded all patients with congenital heart disease or hypertrophic cardiomyopathy due to inherent differences in the pathophysiology and risk of thromboembolic events in these patients.

\section{Statistical Analysis}

Continuous variables are reported using mean \pm SD and compared using the Student $t$ test or rank-sum test as appropriate. Categorical variables are presented as number (\%) and compared using the $\chi^{2}$ test. Univariate logistic regression analysis was performed to identify a parsimonious set of predictors. The final multivariate model was identified based on statistically significant predictors and clinically relevant predictors. Results of these analyses are summarized with odds ratios and the associated 95\% CIs. A $p$ value $<0.05$ was considered statistically significant. All analyses were performed independently by the authors using SAS version 9.4 (Cary, NC, USA).

\section{Results}

During the study period, a total of 7,192 patients with 10,301 DCCVs were enrolled in the database. 765 were excluded due to coexistent congenital heart disease, hypertrophic cardiomyopathy or ESRD requiring dialysis. Of the remaining 6,427, 1,256 (19.5\%) patients had preand post-DCCV SCR available and comprised the study population.

The mean (SD) age of the study population was 70.4 (11.7) years and $67.3 \%$ were male. An average of 13.1 (13.3) SCR values per patient was available during the study period. The mean (SD) time from pre-DCCV SCR to DCCV was 4.9 (12.2) days. The mean (SD) baseline eGFR was $72.1(26.8) \mathrm{mL} / \mathrm{min} / 1.73 \mathrm{~m}^{2}$, and the mean $\mathrm{CHA}_{2} \mathrm{DS}_{2}$-VASc score was 3.4 (1.7) for the entire study cohort. 1,013 (81\%) procedures were performed in an inpatient setting and a total of $1,133(90 \%)$ were successful in restoring sinus rhythm.

During the study period, 131 (10.4\%) patients developed AKI. The mean increase in SCR was $0.5(0.39) \mathrm{mg} /$ $\mathrm{dL}$, and the mean decline in the eGFR was 21.4 (10.78) $\mathrm{mL} / \mathrm{min} / 1.73 \mathrm{~m}^{2}$ for the AKI group. Table 1 chronicles the baseline demographic and clinical characteristics of
28

Cardiorenal Med 2021;11:27-32

DOI: $10.1159 / 000507566$
Grüner-Hegge et al. 
Table 1. Baseline differences between patients with and without AKI after DCCV of AF

\begin{tabular}{|c|c|c|c|}
\hline & $\begin{array}{l}\text { No AKI }(n=1,125) \\
\text { mean }(\mathrm{SD}) \text { or } n(\%)\end{array}$ & $\begin{array}{l}\text { AKI }(n=131) \\
\text { mean }(\mathrm{SD}) \text { or } n(\%)\end{array}$ & $p$ value \\
\hline Age at DCCV, years & $70.15(11.53)$ & $72.58(12.82)$ & 0.004 \\
\hline Female & $348(30.9 \%)$ & $63(48.1 \%)$ & $<0.001$ \\
\hline CKD (eGFR $<60 \mathrm{~mL} / \mathrm{min} / 1.73 \mathrm{~m}^{2}$ at baseline $)$ & $378(33.6 \%)$ & $39(29.8 \%)$ & 0.379 \\
\hline eGFR pre-DCCV (MDRD), $\mathrm{mL} / \mathrm{min} / 1.73 \mathrm{~m}^{2}$ & $71.01(25.35)$ & $81.30(35.53)$ & 0.004 \\
\hline Pre-DCCV serum creatinine, mg/dL & $1.15(0.50)$ & $1.03(0.53)$ & $<0.001$ \\
\hline Estimated onset of present arrhythmia ( $\leq 48 \mathrm{~h})$ & $572(50.8 \%)$ & $84(64.1 \%)$ & 0.046 \\
\hline Pre-cardioversion antiarrhythmic & $134(11.9 \%)$ & $14(10.7 \%)$ & 0.681 \\
\hline Dismissal LMWH & $265(23.6 \%)$ & $25(19.1 \%)$ & 0.250 \\
\hline Beta-blocker & $804(71.5 \%)$ & $102(77.9 \%)$ & 0.122 \\
\hline Calcium channel blocker & $248(22.0 \%)$ & $31(23.7 \%)$ & 0.673 \\
\hline ACE inhibitor & $451(40.1 \%)$ & $47(35.9 \%)$ & 0.351 \\
\hline Diuretic & $632(56.2 \%)$ & $81(61.8 \%)$ & 0.216 \\
\hline Antiarrhythmic & $135(12.0 \%)$ & $14(10.7 \%)$ & 0.660 \\
\hline Spontaneous echo contrast & $253(22.5 \%)$ & $24(18.3 \%)$ & 0.276 \\
\hline LVEF $<35 \%$ & $108(9.6 \%)$ & $14(10.7 \%)$ & 0.691 \\
\hline Pre-cardioversion mean heart rate, bpm & $93.50(22.61)$ & $99.73(21.87)$ & 0.001 \\
\hline Post-cardioversion mean heart rate, bpm & $70.37(16.02)$ & $73.60(20.80)$ & 0.131 \\
\hline Difference pre- to post-HR, bpm & $-23.04(22.66)$ & $-26.32(23.71)$ & 0.127 \\
\hline Median total energy, J & $121.62(30.58)$ & $121.40(27.33)$ & 0.632 \\
\hline Cardioversion successful & $1,020(90.7 \%)$ & $113(86.3 \%)$ & 0.11 \\
\hline Inpatient & $883(78.5 \%)$ & $130(99.2 \%)$ & $<0.001$ \\
\hline $\mathrm{CHA}_{2} \mathrm{DS}_{2}$-VASc score & $3.32(1.68)$ & $3.86(1.66)$ & 0.001 \\
\hline $\mathrm{BPH}$ & $199(25.6 \%)$ & $21(30.9 \%)$ & 0.342 \\
\hline History of CAD & $462(41.1 \%)$ & $59(45.0 \%)$ & 0.383 \\
\hline History of CVA & $69(6.1 \%)$ & $6(4.6 \%)$ & 0.478 \\
\hline History of hypertension & $850(75.6 \%)$ & $102(77.9 \%)$ & 0.560 \\
\hline History of diabetes mellitus & $285(25.3 \%)$ & $39(29.8 \%)$ & 0.272 \\
\hline Smoker (never) & $536(47.6 \%)$ & $68(51.9 \%)$ & 0.652 \\
\hline
\end{tabular}

these patients with and without AKI. Patients with renal injury were more likely to be older women, admitted to the hospital (Fig. 1), with higher HRs and a higher eGFR at baseline. The patient group with AKI had higher burden of cardiovascular risk factors as measured by $\mathrm{CHA}_{2} \mathrm{DS}_{2}$-VASc score $(\mathrm{No}-\mathrm{AKI}=3.32 \pm 1.68$ vs. $\mathrm{AKI}=$ $3.86 \pm 1.66 ; p=0.0007)$.

In the multivariate analysis, AKI was independently associated with inpatient status (OR 26.79; 95\% CI 3.69194.52), $\mathrm{CHA}_{2} \mathrm{DS}_{2}$-VASc score (OR 1.25; 95\% CI 1.111.41), prior use of diuretics (OR 1.59; 95\% CI 1.03-2.46), and absence of CKD (OR 1.61; 95\% CI 1.04-2.49), independent of the success of the DCCV (Table 2). A higher decrease in HR post-DCCV did not show a statistically significant trend towards an increase in AKI in the multivariate analysis (OR 0.99; 95\% CI 0.98-1.0).

None of the patients required acute dialysis during the study outcome period. There were no procedural thromboembolic events.

Renal Dysfunction and Cardioversion

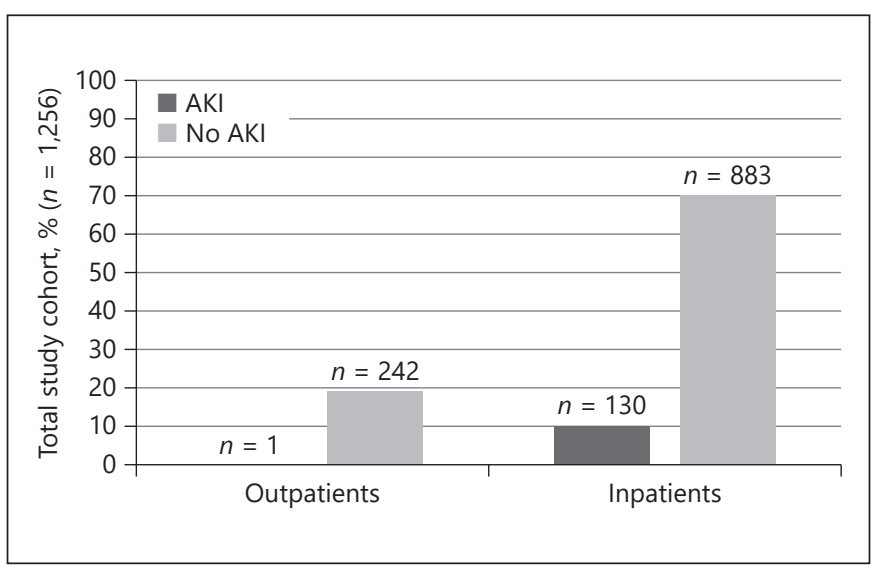

Fig. 1. Difference in AKI incidence between inpatients and outpatients $(p<0.0001)$. 
Table 2. Multivariate predictors of acute kidney injury

\begin{tabular}{lcc}
\hline & Odds ratio $(95 \% \mathrm{CI})$ & $p$ value \\
\hline $\mathrm{CHA}_{2} \mathrm{DS}_{2}$-VASc score & $1.25(1.11-1.41)$ & $<0.001$ \\
Pre-renal normal function $\left(\mathrm{eGFR}>60 \mathrm{~mL} / \mathrm{min} / 1.73 \mathrm{~m}^{2}\right.$ at baseline) & $1.61(1.04-2.49)$ & 0.03 \\
Estimated onset of atrial rhythm $(\leq 48 \mathrm{~h})$ & $1.21(0.75-1.95)$ & 0.43 \\
Difference post-pre heart rate & $0.99(0.98-1.00)$ & 0.05 \\
Inpatient & $26.79(3.69-194.52)$ & 0.001 \\
Successful cardioversion & $0.71(0.39-1.31)$ & 0.27 \\
Spontaneous echo contrast & $0.82(0.50-1.34)$ & 0.42 \\
Pre-DCCV diuretic use & $1.59(1.03-2.46)$ & 0.04 \\
\hline
\end{tabular}

\section{Discussion}

This is, to the best of our knowledge, the largest study to date to evaluate the incidence and predictors of acute renal dysfunction after DCCV for AF. The important findings of this study are: (1) patients are infrequently assessed for renal dysfunction post-DCCV of AF, (2) AKI occurs more frequently in hospitalized individuals, and (3) it is associated with prior diuretic use, higher $\mathrm{CHA}_{2} \mathrm{DS}_{2}$-VASc score and those without underlying renal disease.

Only 19.5\% had pre- and post-SCR measured, which suggests that AKI is an unrecognized complication of DCCV of AF and is not frequently assessed. In a previous report, the incidence of AKI was reported to be $17 \%$ [6]. This relative higher incidence of AKI compared to our study $(10 \%)$ was perhaps related to the differences in the definition of the AKI.

There are several possible mechanisms that can be postulated for the renal dysfunction based on our observations. Acute hemodynamic changes at the time of procedure due to sedation, transient atrial stunning, postDCCV relative bradycardia, severity of acute illness requiring hospitalization, and hypovolemia from fasting state may result in hypoperfusion [18-21]. Mechanical atrial stunning has been implicated in a lack of improvement of cardiac output immediately after DCCV and may also be a contributing factor due to a decrease in renal perfusion [22]. In our study, the incidence of AKI did not show a statistically significant trend towards association with a drop in HR post-DCCV. AF is commonly associated with sinus node dysfunction and is frequently associated with post-DCCV bradyarrhythmias $[8,23]$. Thus, drugs that affect the HR, such as beta-blockers, digoxin, and calcium channel blockers, should be judiciously used after DCCV. Furthermore, the volume status should be optimized before the DCCV with judicious use of diuret- ics to avoid hypovolemia. The presence of CKD is a known risk factor for AKI; however, this relationship is difficult to assess due to the presence of many confounders [24]. In our study, preexisting CKD was protective against AKI. This may be due to the fact that presence of renal disease due to injury of similar or different nature confers protection against future insults by preconditioning as seen in animal models $[25,26]$. In the present study, hospitalized patients were at higher risk for AKI after DCCV of AF despite adjusting for other factors. This patient group may have been further predisposed due to acute illness, difficulty with HR control, thus the need for rhythm control, and higher comorbidity burden.

Electrical cardioversion is known to result in skeletal and myocardial muscle damage, as evidenced by an increase in blood creatinine kinase levels and is related to the cumulative energy delivered [27]. Rhabdomyolysis is a known cause of renal failure [28]. We did not find a significant difference in the incidence of AKI based on the cumulative energy used, suggesting that muscle damage may not be clinically significant to result in AKI.

High $\mathrm{CHA}_{2} \mathrm{DS}_{2}$-VASc score implies significant underlying atherosclerotic disease [29]. There were no acute thromboembolic complications in this study, and AKI was not significantly associated with spontaneous echo contrast in the left atrium, suggesting that large thromboembolism in the setting of adequate anticoagulation is less likely to be the culprit. Micro-thromboembolism has been postulated to play a role in cognitive decline and CKD post-DCCV. Similar mechanisms may play a role in AKI [30]. These potential mechanisms are merely speculative based on observational data and require additional studies.

The change in renal function is most relevant in patients who are on antiarrhythmic drugs that are renally cleared, for example sotalol and dofetilide, parental anticoagulants, for example enoxaparin, and those on direct oral antico-
Grüner-Hegge et al. 
agulants [31]. These drugs may require dose adjustment with a decrease in renal clearance to avoid pro-arrhythmic and bleeding side effects, respectively. Thus, it is imperative to evaluate and closely monitor high-risk patients after DCCV of AF for renal dysfunction.

\section{Study Limitations}

Our findings are best interpreted in the light of the limitations of the study. We only included patients with SCR values, which may have resulted in a selection bias, an inherent limitation of retrospective studies of this nature. We used a previously established and validated definition of AKI based on RIFLE criteria to make the results of the study clinically relevant. This largely validated definition of AKI allowed a longer temporal window to assess outcome for this retrospective study. We did not have data on urine output or continuous hemodynamic monitoring at the time of the DCCV. We did not have data on recent initiation or dose titration of any of the medicines that may have potentially contributed to renal impair- ment. Creatine kinase was not routinely checked to assess the true incidence of rhabdomyolysis.

\section{Conclusions}

$\mathrm{AKI}$ is an unrecognized complication of DCCV of AF and is infrequently assessed. Further prospective studies are required to validate our findings and assess the longterm implications of this complication.

\section{Statement of Ethics}

The Mayo Clinic Institutional Review Board approved the study.

\section{Conflict of Interest Statement}

The authors declare no conflicts of interests.

\section{References}

1 Chugh SS, Blackshear JL, Shen WK, Hammill SC, Gersh BJ. Epidemiology and natural history of atrial fibrillation: clinical implications. J Am Coll Cardiol. 2001 Feb;37(2):371-8.

2 January CT, Wann LS, Alpert JS, Calkins H, Cigarroa JE, Cleveland JC Jr, et al.; ACC/AHA Task Force Members. 2014 AHA/ACC/HRS guideline for the management of patients with atrial fibrillation: executive summary: a report of the American College of Cardiology/American Heart Association Task Force on practice guidelines and the Heart Rhythm Society. Circulation. 2014 Dec;130(23):2071104.

3 Wyse DG, Waldo AL, DiMarco JP, Domanski MJ, Rosenberg Y, Schron EB, et al.; Atrial Fibrillation Follow-up Investigation of Rhythm Management (AFFIRM) Investigators. A comparison of rate control and rhythm control in patients with atrial fibrillation. $\mathrm{N}$ Engl J Med. 2002 Dec;347(23):1825-33.

4 Lown B. Electrical reversion of cardiac arrhythmias. Br Heart J. 1967 Jul;29(4):469-89.

5 Lown B, Perlroth MG, Kaidbey S, Abe T, Harken DE. "Cardioversion" of atrial fibrillation. A report on the treatment of 65 episodes in 50 patients. N Engl J Med. 1963 Aug;269(7):32531.

6 Hellman Y, Cohen MJ, Leibowitz D, Loncar S, Gozal D, Haviv YS, et al. The incidence and prognosis of renal dysfunction following cardioversion of atrial fibrillation. Cardiology. 2013;124(3):184-9.

7 Piccini JP, Fauchier L. Rhythm control in atrial fibrillation. Lancet. 2016 Aug;388(10046): 829-40.
8 Gronberg T, Nuotio I, Nikkinen M et al. Arrhythmic complications after electrical cardioversion of acute atrial fibrillation: the FinCV study. Europace. 2013 Oct;15(10): 1432-5.

9 Lip GY, Tse HF, Lane DA. Atrial fibrillation. Lancet. 2012 Feb;379(9816):648-61.

10 O'Neal WT, Tanner RM, Efird JT, Baber U, Alonso A, Howard VJ, et al. Atrial fibrillation and incident end-stage renal disease: The REasons for Geographic And Racial Differences in Stroke (REGARDS) study. Int J Cardiol. 2015 Apr;185:219-23.

11 Baber U, Howard VJ, Halperin JL, Soliman EZ, Zhang X, McClellan W, et al. Association of chronic kidney disease with atrial fibrillation among adults in the United States: REasons for Geographic and Racial Differences in Stroke (REGARDS) Study. Circ Arrhythm Electrophysiol. 2011 Feb;4(1):26-32.

12 Watanabe H, Watanabe T, Sasaki S, Nagai K, Roden DM, Aizawa Y. Close bidirectional relationship between chronic kidney disease and atrial fibrillation: the Niigata preventive medicine study. Am Heart J. 2009 Oct; 158(4): 629-36.

13 Chaugai S, Sherpa LY, Sepehry AA, Arima H, Wang DW. Effect of RAAS blockers on adverse clinical outcomes in high CVD risk subjects with atrial fibrillation: A meta-analysis and systematic review of randomized controlled trials. Medicine (Baltimore). 2016 Jun; 95(26):e4059.
14 Novo G, Guttilla D, Fazio G, Cooper D, Novo $\mathrm{S}$. The role of the renin-angiotensin system in atrial fibrillation and the therapeutic effects of ACE-Is and ARBS. Br J Clin Pharmacol. 2008 Sep;66(3):345-51.

15 Ammash NM, Phillips SD, Hodge DO, Connolly HM, Grogan MA, Friedman PA, et al. Outcome of direct current cardioversion for atrial arrhythmias in adults with congenital heart disease. Int J Cardiol. 2012 Feb;154(3): 270-4.

16 Bellomo R, Ronco C, Kellum JA, Mehta RL, Palevsky P; Acute Dialysis Quality Initiative workgroup. Acute renal failure - definition, outcome measures, animal models, fluid therapy and information technology needs: the Second International Consensus Conference of the Acute Dialysis Quality Initiative (ADQI) Group. Crit Care. 2004 Aug; 8(4):R204-12.

17 Levey AS, Bosch JP, Lewis JB, Greene T, Rogers N, Roth D; Modification of Diet in Renal Disease Study Group. A more accurate method to estimate glomerular filtration rate from serum creatinine: a new prediction equation. Ann Intern Med. 1999 Mar;130(6):461-70.

18 Sanders NA, Bertolone C, Jetter TL, Wasmund SL, Croci F, Solano A, et al. Restoring sinus rhythm results in blood pressure reduction in patients with persistent atrial fibrillation and a history of hypertension. J Cardiovasc Electrophysiol. 2012 Jul;23(7):722-6.

19 Jacob M, Chappell D. Effects of perioperative fasting on haemodynamics and intravascular volumes. Best Pract Res Clin Anaesthesiol. 2012 Dec;26(4):421-30. 
20 Grimm RA, Stewart WJ, Maloney JD, Cohen GI, Pearce GL, Salcedo EE, et al. Impact of electrical cardioversion for atrial fibrillation on left atrial appendage function and spontaneous echo contrast: characterization by simultaneous transesophageal echocardiography. J Am Coll Cardiol. 1993 Nov;22(5): 1359-66.

21 Irani WN, Grayburn PA, Afridi I. Prevalence of thrombus, spontaneous echo contrast, and atrial stunning in patients undergoing cardioversion of atrial flutter. A prospective study using transesophageal echocardiography. Circulation. 1997 Feb;95(4):962-6.

22 Khan IA. Atrial stunning: basics and clinical considerations. Int J Cardiol. 2003 Dec;92(23):113-28.

23 John RM, Kumar S. Sinus Node and Atrial Arrhythmias. Circulation. 2016 May;133(19): 1892-900.
24 Singh P, Rifkin DE, Blantz RC. Chronic kidney disease: an inherent risk factor for acute kidney injury? Clin J Am Soc Nephrol. 2010 Sep;5(9):1690-5.

25 Bonventre JV. Kidney ischemic preconditioning. Curr Opin Nephrol Hypertens. 2002 Jan;11(1):43-8.

26 Goldfarb M, Rosenberger C, Abassi Z, Shina A, Zilbersat F, Eckardt KU, et al. Acute-onchronic renal failure in the rat: functional compensation and hypoxia tolerance. Am J Nephrol. 2006;26(1):22-33.

27 Jakobsson J, Odmansson I, Nordlander R. Enzyme release after elective cardioversion. Eur Heart J. 1990 Aug;11(8):749-52.

28 Cavusoglu Y, Entok E, Gorenek B, Kudaiberdieva G, Unalir A, Goktekin O, et al. Reversible myoglobinuric renal failure following rhabdomyolysis as a rare complication of cardioversion. Pacing Clin Electrophysiol. 2003 Feb;26(2 Pt 1):645-6.
29 Chade AR, Lerman A, Lerman LO. Kidney in early atherosclerosis. Hypertension. 2005;45: 1042-9.

30 Corrao S, Argano C, Nobili A, Marcucci M, Djade CD, Tettamanti M, et al.; REPOSI Investigators. Brain and kidney, victims of atrial microembolism in elderly hospitalized patients? Data from the REPOSI study. Eur J Intern Med. 2015 May;26(4):243-9.

31 Boriani G, Savelieva I, Dan GA, et al. Chronic kidney disease in patients with cardiac rhythm disturbances or implantable electrical devices: clinical significance and implications for decision making-a position paper of the $\mathrm{Eu}$ ropean Heart Rhythm Association endorsed by the Heart Rhythm Society and the Asia Pacific Heart Rhythm Society. Europace. 2015 Aug;17(8):1169-96. 